\title{
Frontières
}

Vincent Paris, Zombies-Sociologie des morts-vivants, XYZ, 2013

Geneviève Pigeon

Volume 25, numéro 2, 2013

URI : https://id.erudit.org/iderudit/1024948ar

DOI : https://doi.org/10.7202/1024948ar

Aller au sommaire du numéro

Éditeur(s)

Université du Québec à Montréal

ISSN

1916-0976 (numérique)

Découvrir la revue

Citer ce compte rendu

Pigeon, G. (2013). Compte rendu de [Vincent Paris, Zombies-Sociologie des morts-vivants, XYZ, 2013]. Frontières, 25(2), 147-148.

https://doi.org/10.7202/1024948ar

Ce document est protégé par la loi sur le droit d'auteur. L'utilisation des services d'Érudit (y compris la reproduction) est assujettie à sa politique d'utilisation que vous pouvez consulter en ligne.

https://apropos.erudit.org/fr/usagers/politique-dutilisation/
Cet article est diffusé et préservé par Érudit.

Érudit est un consortium interuniversitaire sans but lucratif composé de l’Université de Montréal, l'Université Laval et l'Université du Québec à Montréal. Il a pour mission la promotion et la valorisation de la recherche. https://www.erudit.org/fr/ 
part, avec le cadre post-apocalyptique que Nick Muntean trace un parallèle en comparant L'aube des morts-vivants (1978) de Romero au film de Stanley Kramer Sur la plage (1959) d'après le roman éponyme de Nevil Shute. Steven Zani et Kevin Meaux abordent I'œuvre d'un autre grand artisan du film de zombies, le cinéaste italien Lucio Fulci, alors que Bernice Murphy et Sorcha Ní Fhlainn étudient respectivement les figures du zombie banlieusard et du soldat zombifié.

La troisième et dernière section de Better off dead met l'accent sur les transformations qu'a subies le mythe du zombie au tournant du millénaire. Lynn Pifer commente I'assimilation du zombie au type du fainéant de la génération $X$ dans la comédie Shaun of the dead. Peter Dendle s'intéresse à la génération suivante pour élucider un surprenant paradoxe: les enfants du millénaire, habitués aux sollicitations continues, spontanées et effrénées des jeux vidéo et des médias sociaux, sont de grands adeptes du zombie en qui ils voient un reflet de leur identité et de leurs valeurs. Margo Collins et Elson Bond montrent que les deux incarnations du zombie contemporain, le zombie comique et le zombie menaçant, se chevauchent pour énoncer des visions d'espoir et en appeler à une ré-humanisation de I'homme. Sarah Juliet Lauro, dans la dernière étude de l'ouvrage, se tourne vers l'art conceptuel de Jillian Mcdonald, dont l'installation Zombie Loop lui paraît s'apparenter aux pratiques de I'Internationale situationniste dans les années 1960.

À travers treize études savantes et originales, Better off dead propose un fascinant survol de l'évolution du zombie à l'heure des nouvelles biotechnologies et du transhumanisme. Une grande diversité d'angles sont couverts, depuis les origines folkloriques et cinématographiques du monstre jusqu'à ses plus surprenantes métamorphoses dans la culture populaire. On s'explique mal toutefois l'absence d'un grand artisan de la Renaissance zombie depuis le début des années 2000: Robert Kirkman, créateur de la saga graphique The Walking Dead. En ne lui faisant aucunement allusion, Christie et Lauro se rendent coupables d'une singulière omission.

\section{Vincent Paris}

\section{Zombies-Sociologie des morts-vivants}

$\mathrm{XYZ}, 2013$

\section{Geneviève Pigeon, Ph. D.}

Chargée de cours, Département de sciences des religions, Université du Québec à Montréal

Titulaire d'une maîtrise en science politique et d'une scolarité de doctorat en sociologie, Vincent Paris aborde dans Zombies - Sociologie des morts-vivants un phénomène de société devenu incontournable. L'approche proposée par l'auteur, également professeur de sociologie au cégep, se veut avant tout ludique et légère. À cet égard, on peut affirmer 
qu'il atteint son objectif, soit celui d'explorer «un phénomène social qui, au cours des dernières années, a pris de plus en plus d'ampleur».

Divisé en quatre parties, Zombies - Sociologie des morts-vivants s'intéresse dans un premier temps à la vie des morts-vivants, à leurs habitudes, leurs besoins, leurs comportements sociaux et individuels et ce, en s'adressant au lecteur comme si ce dernier devenait subitement un mortvivant. Ce premier chapitre fait en quelque sorte un survol de la littérature destinée aux zombies ( $\mathrm{si}$ si) tout en ouvrant un aspect sociétal spectaculaire de la popularité des zombies: les marches de morts-vivants.

L'auteur enchaîne ensuite avec des chapitres portant sur les zombies dans l'actualité, sur leurs origines et sur la popularité du phénomène. Si la volonté initiale de produire un livre accessible, sympathique et simple est louable et bien accomplie, l'ouvrage présente un certain nombre de lacunes qui l'empêcheront de constituer une référence utile en matière de manifestations culturelles populaires. En effet, si les différents apports de la sociologie sont plutôt bien annoncés et amorcés, et ce dans la mesure où cette science s'intéresse à la collectivité et à ses fonctionnements, Vincent Paris peine à convaincre le lecteur de la pertinence de son analyse du zombie d'autant plus que ce dernier est présenté comme un être asocial (p. 61).

Outre certains enchaînements plus ou moins réussis et certains flottements quant à l'objet précis de l'étude (le zombie ou la «vraie» société qui I'a fait naître et évoluer en tant qu'objet social et culturel?), la partie traitant des origines des zombies pose un certain nombre de problèmes assez importants. S'il est clairement expliqué par l'auteur que le vaudou haïtien ne saurait seul être à la source de ce phénomène aujourd'hui universel, il est difficile de concevoir comment le recours à quelques passages bibliques peut éclairer davantage la situation.

On pourra opposer à ces commentaires l'ambition vulgarisatrice de l'ouvrage, ambition certes louable, mais qui résiste mal à une abondance de citations peu ou pas référencées. Peut-être aurait-il mieux valu demeurer plus près de la sphère sociologique et laisser de côté l'aspect historique et religieux de l'objet «zombie / mort-vivant». Ce faisant, certains aspects de l'analyse du phénomène auraient pu être mis de côté pour une étude future. Ainsi, I'histoire complexe des infanticides n'aurait pas été expédiée en quelques lignes discutables et le mystère de l'eucharistie aurait été traité avec le respect symbolique qui lui est dû.

Le problème riche et complexe de la popularité des zombies demeure un objet de recherche fascinant, et I'enthousiasme contagieux de Vincent Paris est d'autant plus frustrant que Zombies - Sociologie des mortsvivants donne l'impression d'être le brouillon d'une recherche plus vaste et extrêmement fertile. Souhaitons qu'il nous revienne avec une étude à la hauteur de ses intuitions, par ailleurs fort intéressantes. 